\title{
Compound Heterozygous Mutations in TMC1 and MYO15A Are Associated with Autosomal Recessive Nonsyndromic Hearing Loss in Two Chinese Han Families
}

\author{
Pengcheng Xu, ${ }^{1,2,3}$ Jun $X u \mathbb{D}^{1,2,3} \mathrm{Hu}^{\text {Peng, }}{ }^{4}$ and Tao Yang $\mathbb{D}^{1,2,3}$ \\ ${ }^{1}$ Department of Otorhinolaryngology-Head and Neck Surgery, Shanghai Ninth People's Hospital, Shanghai Jiao Tong University \\ School of Medicine, Shanghai, China \\ ${ }^{2}$ Ear Institute, Shanghai Jiao Tong University School of Medicine, Shanghai, China \\ ${ }^{3}$ Shanghai Key Laboratory of Translational Medicine on Ear and Nose Diseases, Shanghai, China \\ ${ }^{4}$ Department of Otolaryngology-Head and Neck Surgery, Changzheng Hospital, Second Military Medical University, Shanghai, China
}

Correspondence should be addressed to Tao Yang; taoyang@sh-jei.org

Received 22 April 2020; Revised 12 June 2020; Accepted 6 July 2020; Published 1 August 2020

Academic Editor: Renjie Chai

Copyright (c) 2020 Pengcheng Xu et al. This is an open access article distributed under the Creative Commons Attribution License, which permits unrestricted use, distribution, and reproduction in any medium, provided the original work is properly cited.

\begin{abstract}
Genetic hearing loss is a common sensory disorder, and its cause is highly heterogeneous. In this study, by targeted next-generation sequencing of 414 known deafness genes, we identified compound heterozygous mutations p.R34X/p.M413T in TMC1 and p.S3417del/p.R1407T in MYO15A in two recessive Chinese Han deaf families. Intrafamilial cosegregation of the mutations with the hearing phenotype was confirmed in both families by the Sanger sequencing. Auditory features of the affected individuals are consistent with that previously reported for recessive mutations in TMC1 and MYO15A. The two novel mutations identified in this study, p.M413T in TMC1 and p.R1407T in MYO15A, are classified as likely pathogenic according to the guidelines of ACMG. Our study expanded the mutation spectrums of TMC1 and MYO15A and illustrated that genotype-phenotype correlation in combination with next-generation sequencing may improve the accuracy for genetic diagnosis of deafness.
\end{abstract}

\section{Introduction}

Congenital hearing impairment is a common birth defect worldwide, occurring in approximately 1-2 per 1000 infants. With increasing age, the prevalence continues to rise to 2.7 per 1000 before age five and 3.5 per 1000 through adolescence [1]. To date, more than 100 genes have been reported to be associated with nonsyndromic hearing loss (NSHL), including 76 autosomal recessive nonsyndromic hearing loss (ARNSHL) genes, 48 autosomal dominant nonsyndromic hearing loss (ADNSHL) genes, and 5 X-linked nonsyndromic hearing loss genes (Hereditary Hearing Loss Homepage; https://hereditaryhearingloss.org/, updated in January 2020). Hair cells (HCs) in the cochlea mainly function in converting the sound mechanical waves into the electric neural signals [2-4] which make it extremely critical for the hearing ability. Many previous studies have shown that HCs can be damaged due to genetic factors, ototoxic drugs, noise, inflammation, or aging, among which genetic account for $50 \%$ of the HC malfunction [5-11].

The TMC1 gene is located on chromosome 9q21 and contains 24 exons that encodes a 760 amino acid membrane protein $\mathrm{TMC1}$ with six transmembrane domains $[12,13]$. TMC1 is a pore-forming subunit of the mechanotransduction complex that is predicted to have transmembrane domains with intracellular $\mathrm{N}$ and $\mathrm{C}$ termini and one conserved TMC domain [14]. TMC1 is expressed in the mouse inner ear and has been suggested to involve in the functional maturation and survival of cochlear HCs [12]. It has been reported that mutations in TMC1 may cause both prelingual profound autosomal recessive deafness DFNB7/11 and postlingual progressive autosomal dominant deafness DFNA36 [13]. To date, more than 60 mutations in $T M C 1$ are reported worldwide [15], with the recessive mutations predominantly associated with prelingual severe-to-profound hearing loss $[15,16]$. 


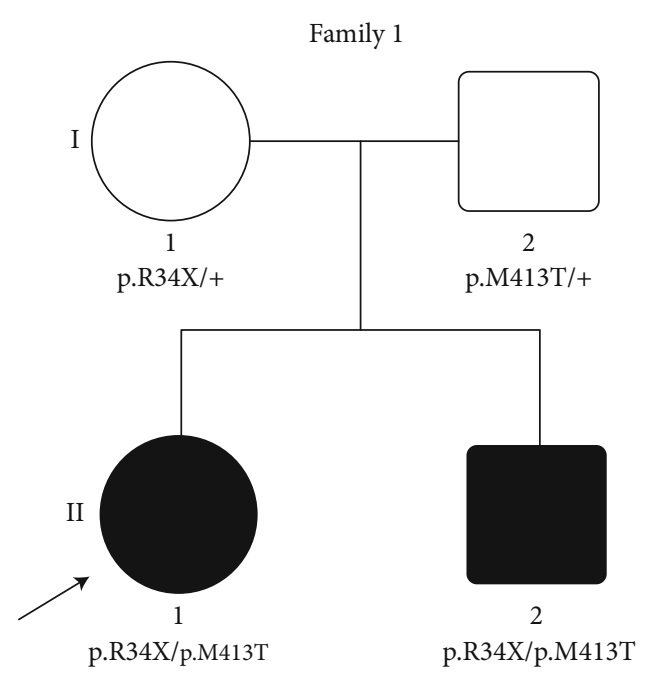

(a)

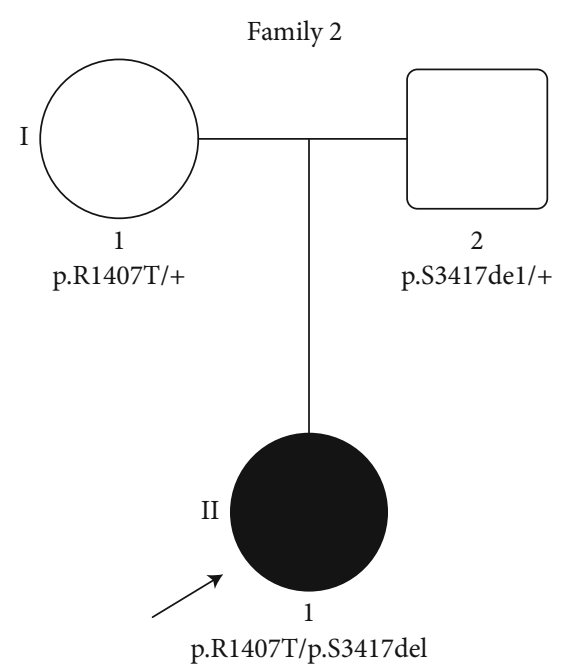

(b)

Figure 1: Pedigrees and genotypes of Family 1 (a) and Family 2 (b).

The HC stereocilia is critical to maintain the function of HC $[17,18]$. The MYO15A gene is located at chromosome 17 p11.2 and contains 66 coding exons, which encodes an unconventional myosin protein Myosin XVA [19]. Myosin $\mathrm{XVA}$ is a large actin-based motor protein. In cochlear hair cells, it is critical for elongation and differentiation of the stereocilia [20]. Myosin XVA displays an important role in the mechanotransduction of cochlear hair cells. Myosin XVA through its carboxy-terminal PDZ-ligand interacts with the third PDZ domain of whirlin, and then delivers whirlin to the tips of stereocilia [21]. MYO15A mutations are responsible for congenital deafness DFNB3 in human and cochleovestibular dysfunction in shaker 2 mice which shows abnormally short stereocilia bundles and diminished staircase $[20,21]$. It is one of the most common causes of ADNSHL in Mideast countries due to prevalent consanguineous marriage $[22,23]$, with majority associated with prelingual severe-to-profound hearing loss and mutations in exon 2 leading to a milder auditory phenotype [23].

In this study, we presented the clinical characterization and genetic analysis of two Chinese Han families affected by ARNSHL. Using targeted next-generation sequencing of 414 known deafness genes, we identified compound heterozygous mutations in TMC1 and MYO15A as the genetic causes of the hearing loss in those families.

\section{Materials and Methods}

2.1. Subjects and Clinical Evaluations. This study included two nuclear Chinese Han recessive deaf families: Family 1 (Figure 1(a)) and Family 2 (Figure 1(b)). All affected family members underwent clinical evaluation in the Department of Otolaryngology-Head and Neck Surgery, Shanghai Ninth People's Hospital, Shanghai Jiao Tong University School of Medicine, Shanghai, China. The evaluation included a complete medical history interview and a comprehensive physical examination including otoscopic examination to exclude hearing loss due to infections, trauma, or other environmen- tal factories. Middle ear function was evaluated through tympanometry, and the function of the outer hair cells of the cochlea was evaluated by distortion production otoacoustic emissions (DPOAE). Pure-tone audiometry (PTA) was calculated as the average of the hearing threshold of patients at $500,1,000,2,000$, and $4,000 \mathrm{~Hz}$. The degree of hearing loss was defined as mild $(26-40 \mathrm{~dB} \mathrm{HL})$, moderate $(41-55 \mathrm{~dB}$ $\mathrm{HL})$, moderately severe (56-70 dB HL), severe (71-90 dB $\mathrm{HL})$, and profound $(>90 \mathrm{~dB} \mathrm{HL})$. Hearing thresholds reported in this study were averaged air-conducted puretone thresholds of each side. Tandem gait and the Romberg testing were performed for vestibular function examination. Computerized tomography (CT) scan of the temporal bone was carried out to assess the development of the anatomical structures of the middle and inner ear for the available subjects. This study was approved by the ethnic committee of Shanghai Ninth People's Hospital. Written informed consents were obtained from each participant or from parents of the young subject.

2.2. Mutation Identification. The genomic DNA of peripheral blood was extracted from all subjects using a Blood DNA kit according to the standard protocol (QIAamp DNA Blood Mini Kit, QIAGEN, Shanghai). Targeted next-generation sequencing was performed as previously reported [24, 25]. The exons, splicing sites, and flanking intronic region of 414 known deafness-related genes (Table S1) were captured by a customized capture assay (MyGenostics, Beijing, China). Candidate pathogenic mutations were defined as nonsynonymous (including nonsense, missense, splice-site, and indels) variants that had allele frequencies under 0.01 in the 1000 Genomes database, the dbSNP database, the Exome Aggregation Consortium database (ExAC), and data from 200 Chinese Han normal-hearing control individuals. The potential pathogenic effects of candidate mutations were evaluated by in silico tools Mutation Taster, SIFT, and PolyPhen2. Cosegregation of the disease phenotype and the causative mutation was confirmed in all family members by 


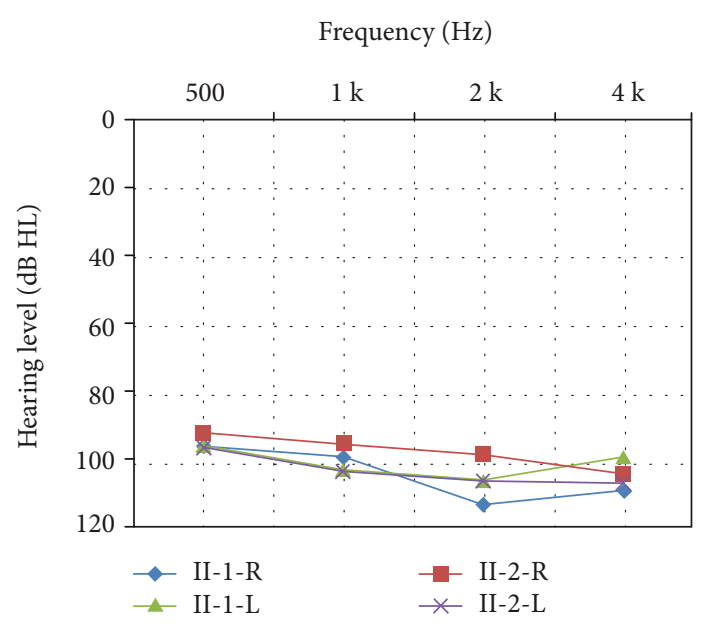

(a)

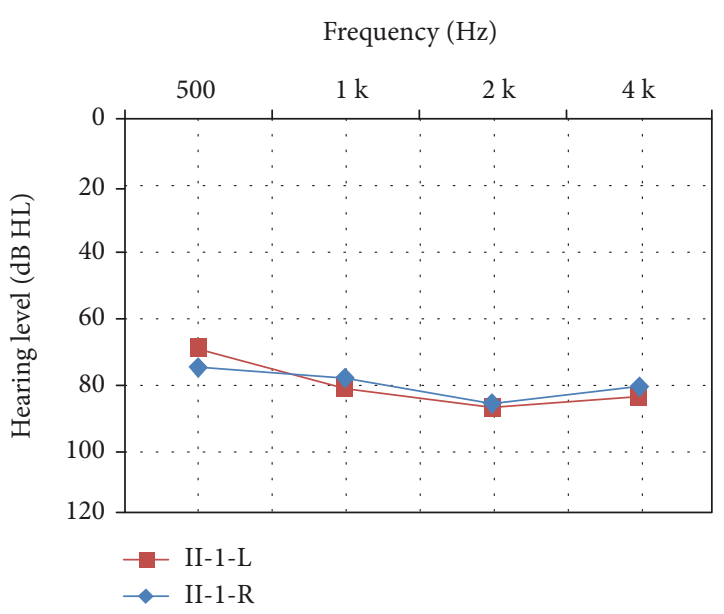

(b)

Figure 2: Audiograms of the affected members of Family 1 (a) and Family 2 (b).

PCR amplification and the Sanger sequencing. Pathogenicity of the mutations were classified following the guidelines of ACMG 2015 [26].

\section{Results}

\subsection{Clinical Characterization}

3.1.1. Family 1. Family 1 has two affected siblings born to two normal-hearing parents (Figure 1(a)). The proband II1 was a 26-year-old female with congenital sensorineural hearing loss. Tympanogram indicated normal function of the middle ear. Bilateral DPOAE were absent. Both II-1 and her younger brother II-2 suffered from bilateral, profound hearing impairment with PTA thresholds above $90 \mathrm{~dB}$ HL (Figure 2(a)). Tandem gait and the Romberg testing displayed no symptoms of vestibular dysfunction. Temporal bone CT scans showed no obvious abnormalities. No apparent additional syndromic features were found.

3.1.2. Family 2. The proband II-1 of Family 2 (Figure 1(b)) was a 10-year-old girl, who suffered from prelingual bilateral hearing impairment. Auditory examination and PTA showed the sensorineural hearing loss is severe (Figure 2(b)). Tympanogram displayed a type A curve, indicating normal function of the middle ear. Bilateral DPOAE were absent, and no vestibular dysfunction was recorded for the proband. No other abnormality was discovered by the medical history and physical examination.

3.2. Mutation Analysis. Targeted next-generation sequencing of 414 known deafness genes was performed for the probands Family1-II-1 and Family2-II-1. A total of 9 and 13 candidate variants were identified, respectively (Table S2). In Family 1, compound heterozygous mutations c.100C $>\mathrm{T}$ (p.R34X) and c.1238T>C (p.M413T) in TMC1 (NM_138691) were identified as the only candidate mutations consistent with the recessive inheritance. The Sanger sequencing revealed that the mutations cosegregated with the hearing phenotype in Family 1, as the unaffected parents, were heterozygous carriers of single mutations p.R34X (mother
I-1) and p.M413T (father I-2), while the affected siblings both had compound heterozygous mutations (Figure 3(a)). These two mutations were not detected in 200 Chinese Han normal-hearing controls and are not present in 1000 Genomes and ExAC databases. The p.R34X mutation with minor allele frequency (MAF) of 0.0002 in ExAC has been previously detected in many patients from Pakistan, Iran, Turkey, and Tunisia but is relatively rare in China $[16,27-$ 29]. On the other hand, while the p.M413T mutation is novel. Based on the ACMG guidelines, the p.R34X and p.M413T mutations in TMC1 were classified as pathogenic $(\mathrm{PVS} 1+\mathrm{PS} 1+\mathrm{PM} 2+\mathrm{BS} 2)$ and likely pathogenic (PM2+PM3 $+\mathrm{PP} 3)$, respectively.

In Family 2, compound heterozygous variants c.10245 10247delCTC (p.S3417del) and c.4220G >C (p.R1407T) in MYO15A (NM_016239) were considered the only candidate pathogenic variants consistent with the recessive inheritance. The Sanger sequencing confirmed that the mutations cosegregated with the hearing phenotype in Family 2, as the unaffected parents were heterozygous carriers of single mutations p.R1407T (mother I-1) and p.S3417del (father I-2) (Figure 4(a)). These two mutations were not detected in 200 Chinese Han normal-hearing controls and are not present in 1000 Genomes and ExAC databases. The p.S3417del mutation with MAF of 0.000016 in ExAC has been previously reported to cause autosomal recessive hearing loss in Japanese and Korean patients, but not in the Chinese population [30, 31]. The p.R1407T mutation is novel. Based on the ACMG guidelines, the p.S3417del and p.R1407T mutations in MYO15A were classified as pathogenic (PS1+PM2+PM3+PM4) and likely pathogenic (PM2 $+\mathrm{PM} 3+\mathrm{PP} 3)$, respectively.

\section{Discussion}

Recessive hearing loss accounts for the majority (80\%) of genetic hearing loss [32]. Among many genes responsible for ARSNHL, mutations in GJB2 are the most frequent causes $[33,34]$, followed by that in SLC26A4, TMC1, and MYO15A especially in Middle East countries where 




(a)

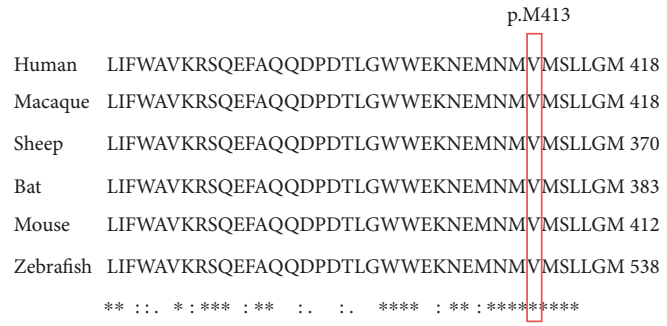

(b)

Figure 3: (a) The Sanger sequencing results of the p.R34X and p.M413T mutations in TMC1 in Family 1. (b) Multispecies sequence alignment of the M413 residue.

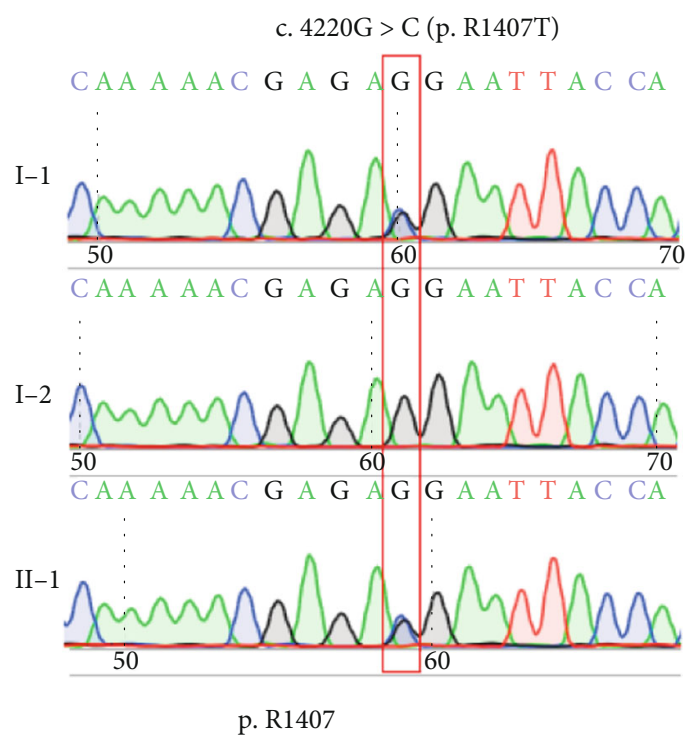

Human VFQAKNERNYHIFYELLAGLPAQLSLQE 1431 Macaque VFQAKNERNYHIFYELLAGLPAQLSLQE 1430 Sheep VFQAKNERNYHIFYELLAGLPAQLSLQE 1327 Bat VFQAKNERNYHIFYELLAGLPAQLSLQE 1145 Mouse VFQAKNERNYHIFYELLAGLPAQLSLQE 1415 Zebrafish VFQAKNERNYHIFYELLAGLPAQLSLQE 2039 ****:: $: * * * * * * * * * * *: * * * * * *: *: *: * * *:$

(a)

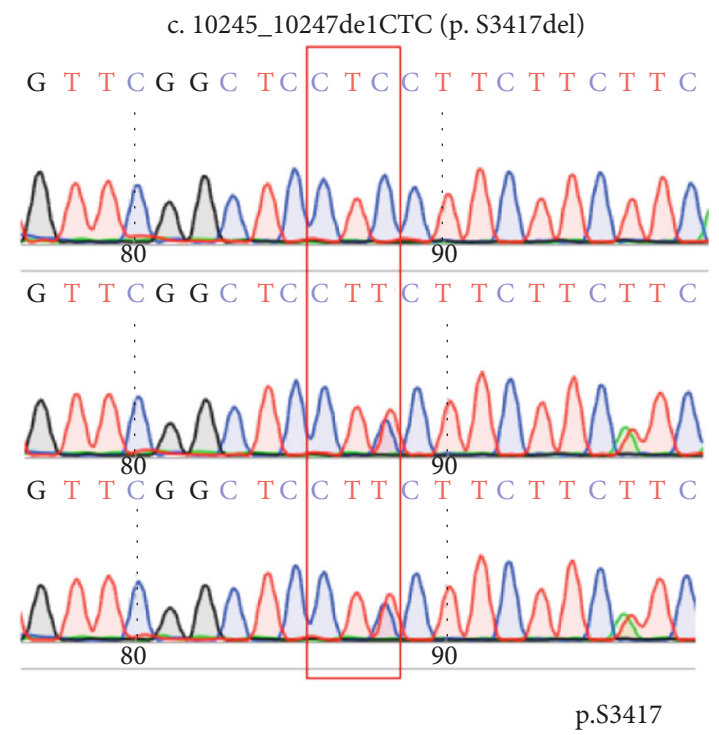

Human QTQALSPHQARAQFLGLLSALPMFGSSFFF 3420 Macaque QTQALSPHQARAQFLGLLSALPMFGSSFFF 3418 Sheep QTQALSPHQARAQFLGLLSALPMFGSSFFF 3275 Bat QTQALSPHQARAQFLGLLSALPMFGSSFFF 3146 Mouse QTQALSPHQARAQFLGLLSALPMFGSSFFF 3410 Zebrafish QTQALSPHQARAQFLGLLSALPMFGSSFFF 4005 $* * * * * * * * *: * * * * * * . * * *$.

(b)

FIgURe 4: (a) The Sanger sequencing results of the p.S3417del and p.R1407T mutations in MYO15A in Family 2. (b) Multispecies sequence alignment of the S3417 and R1407 residues.

consanguineous marriage is common $[15,22,35]$. On the contrary, in China where consanguineous marriage is far less frequent, recessive mutations in TMC1 and MYO15A were not as extensively reported in the literature.

In Family 1, we identified compound heterozygous mutations p.R34X and p.M413T in TMC1. The p.R34X mutation is the most common TMC1 mutation in Pakistan [36]. Using polymorphic markers, Ben Said et al. showed that this non- sense mutation is an old founder mutation emerged between the years 1075 and 1900 along with the third Hadhramaut population movements [27]. This nonsense mutation is predicted to produce a prematurely truncated protein and is associated with congenital, severe-to-profound deafness [13, 27]. The p.M413T mutation identified in this study has not been previously reported. It is predicted as deleterious by computational tools PolyPhen2 and SIFT. The p.M413T 


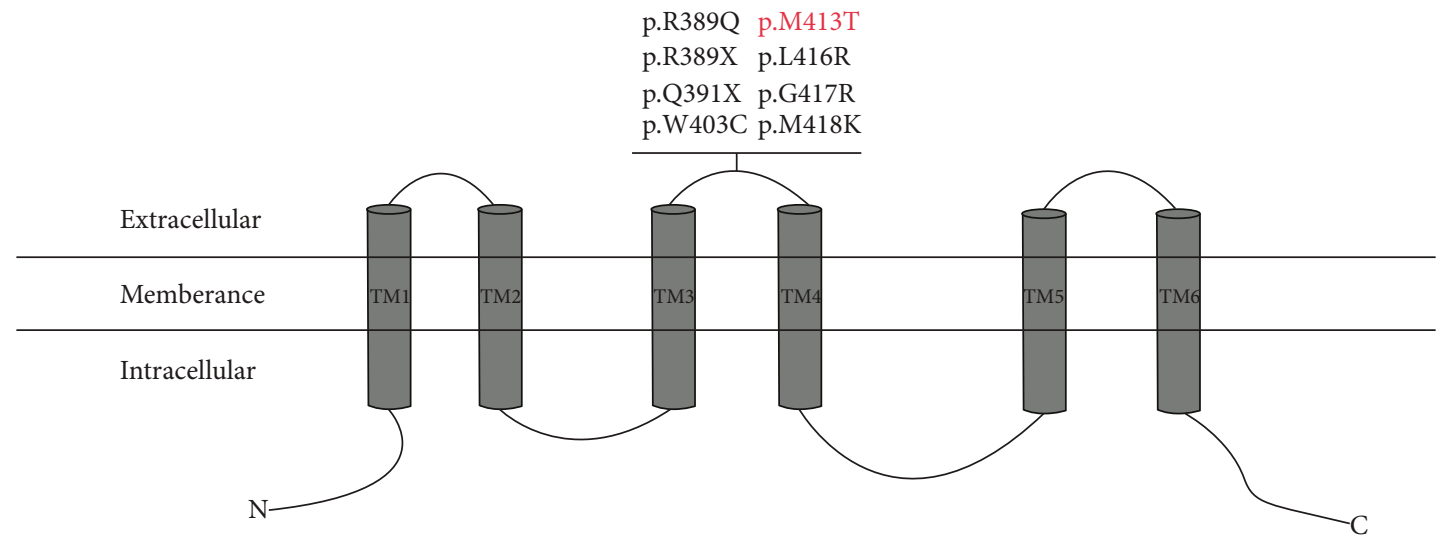

(a)

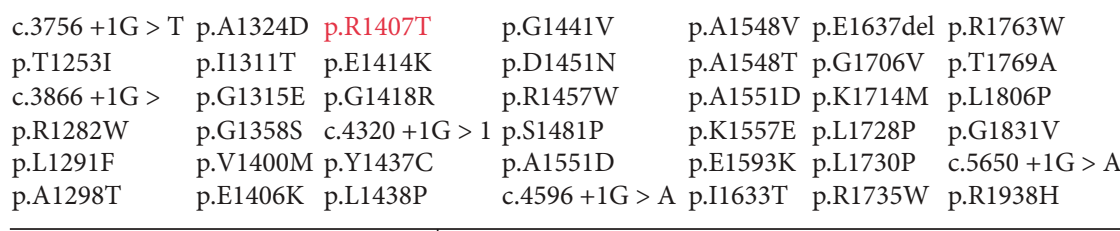

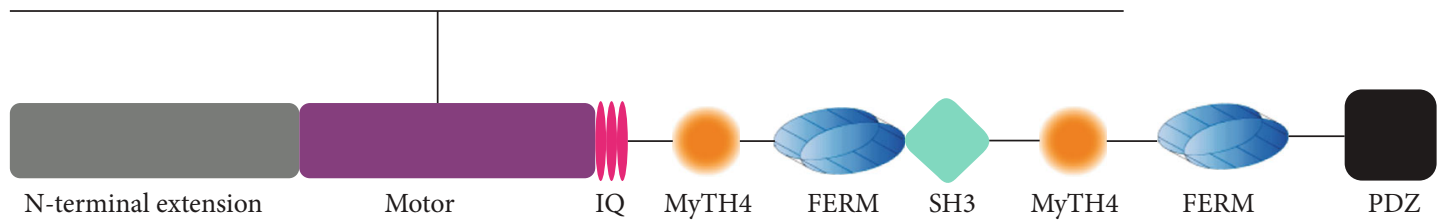

(b)

Figure 5: (a) Transmembrane domain structure of TMC1 and mutations in the second extracellular loop of TMC1. The novel p.M413T mutation identified in this study is in red. (b) Protein structure of Myosin XVA and missense mutations in the motor domain. The novel p.R1407T mutation identified in this study is in red.

mutation is located in the second extracellular loop between the third and fourth transmembrane domains of TMC1, and the methionine 413 residue is well conserved among different species (Figure 3(b)). At least seven mutations in the second extracellular loop, including five missense mutations, have already been associated with ARNSHL and ADNSHL (Figure 5(a)), suggesting an important role of this particular region in the inner ear function of TMC1.

In Family 2, we identified compound heterozygous mutations p.S3417del and p.R1407T in MYO15A. The p.S3417del mutation deletes a serine 3417 residue at the second FERM domain of Myosin XVA. The FERM domain is a protein-binding domain important in cargo transport and cytoplasmic protein connection to the membrane [37, 38]. This mutation has been previously reported in Japanese and Korean deaf patients but not in the Chinese population $[30,31]$. The novel p.R1407T mutation identified in this study is predicted as deleterious by computational tools PolyPhen2 and SIFT. This mutation is located in the motor domain of Myosin XVA, which is next to the long $\mathrm{N}$ terminal extension and is highly conserved among different species (Figure 4(b)). To date, more than 40 missense mutations in the motor domain of MYO15A have been associated with ARSNHL (Figure 5(b)). The motor domain is essential for ATP activity and possesses two binding sites for actin and ATP, which can produce force to move the actin filaments. In the mouse model, MYO15A mutation in the motor domain results shorter stereocilia with an abnormal staircase structure [39].

Considering the high degree of genetic heterogeneity, the next-generation sequencing (NGS) technology has been proven an effective method for genetic testing of hearing loss in recent years. However, previous studies have showed that NGS in deaf patients, especially the sporadic cases, may detect a significant amount of rare, nonsynonymous variants with unknown functional significance and sometimes even results in false-positive diagnosis [25]. In this study, we obtained a detailed hearing phenotype for all patients, which is consistent with those from previous reports for ARSNHL patients with recessive TMC1 and MYO15A mutations. Our data suggested that the genotype-phenotype correlation may facilitate more accurate genetic diagnosis of deafness in such cases.

\section{Conclusions}

Compound heterozygous mutations p.R34X/p.M413T in TMC1 and p.S3417del/p.R1407T in MYO15A were identified as the pathogenic causes of ARSNHL in two Chinese Han families. Our results expanded the mutation spectrum of those two genes and showed that NGS in combination with genotype-phenotype correlation may provide a more accurate diagnosis for genetic deafness. 


\section{Data Availability}

The data used to support the findings of this study are available from the corresponding authors upon request.

\section{Conflicts of Interest}

The authors declare no conflicts of interests.

\section{Authors' Contributions}

Pengcheng $\mathrm{Xu}$, Jun $\mathrm{Xu}$, and $\mathrm{Hu}$ Peng contributed equally to this work.

\section{Acknowledgments}

This research was supported by grants from the Shanghai Municipal Education Commission-Gaofeng Clinical Medicine Grant (20152519 to TY), Science Project of Shanghai Municipal Commission of Health and Family Planning (201540173 to HP), and National Natural Science Foundation of China (81702643 to HP).

\section{Supplementary Materials}

Table S1: the 414 deafness-related genes sequenced by targeted NGS. Table S2: candidate mutations identified in probands F1-II-1 and F2-II-1 by targeted NGS. (Supplementary Materials)

\section{References}

[1] C. C. Morton and W. E. Nance, "Newborn hearing screeninga silent revolution," The New England Journal of Medicine, vol. 354, no. 20, pp. 2151-2164, 2006.

[2] Y. Liu, J. Qi, X. Chen et al., "Critical role of spectrin in hearing development and deafness," Science Advances, vol. 5, no. 4, 2019.

[3] Y. Wang, J. Li, X. Yao et al., "Loss of CIB2 causes profound hearing loss and abolishes mechanoelectrical transduction in mice," Frontiers in molecular neuroscience, vol. 10, 2017.

[4] C. Zhu, C. Cheng, Y. Wang et al., "Loss of ARHGEF6 causes hair cell stereocilia deficits and hearing loss in mice," Frontiers in molecular neuroscience, vol. 11, 2018.

[5] Z. H. He, S. Y. Zou, M. Li et al., "The nuclear transcription factor FoxG1 affects the sensitivity of mimetic aging hair cells to inflammation by regulating autophagy pathways," Redox biology, vol. 28, p. 101364, 2020.

[6] Y. Zhang, W. Li, Z. He et al., "Pre-treatment with fasudil prevents neomycin-induced hair cell damage by reducing the accumulation of reactive oxygen species," Frontiers in Molecular Neuroscience, vol. 12, 2019.

[7] R. Chai, S. Gao, C. Cheng et al., "Blebbistatin inhibits neomycin-induced apoptosis in hair cell-like HEI-OC-1 cells and in cochlear hair cells," Frontiers in Cellular Neuroscience, vol. 13, 2019.

[8] L. Liu, Y. Chen, J. Qi et al., "Wnt activation protects against neomycin-induced hair cell damage in the mouse cochlea," Cell death \& disease, vol. 7, no. 3, 2016.

[9] W. Liu, X. Xu, Z. Fan et al., "Wnt signaling activates TP53induced glycolysis and apoptosis regulator and protects against cisplatin-induced spiral ganglion neuron damage in the mouse cochlea," Antioxidants \& redox signaling, vol. 30, no. 11, pp. 1389-1410, 2019.

[10] H. Li, Y. Song, Z. He et al., "Meclofenamic acid reduces reactive oxygen species accumulation and apoptosis, inhibits excessive autophagy, and protects hair cell-like HEI-OC1 cells from cisplatin-induced damage," Frontiers in cellular neuroscience, vol. 12, 2018.

[11] S. Zhang, Y. Zhang, Y. Dong et al., "Knockdown of Foxg1 in supporting cells increases the trans-differentiation of supporting cells into hair cells in the neonatal mouse cochlea," Cellular and Molecular Life Sciences, vol. 77, no. 7, pp. 1401-1419, 2020.

[12] Y. Kawashima, G. S. G. Géléoc, K. Kurima et al., "Mechanotransduction in mouse inner ear hair cells requires transmembrane channel-like genes," The Journal of Clinical Investigation, vol. 121, no. 12, pp. 4796-4809, 2011.

[13] K. Kurima, L. M. Peters, Y. Yang et al., "Dominant and recessive deafness caused by mutations of a novel gene, TMC1, required for cochlear hair-cell function," Nature Genetics, vol. 30, no. 3, pp. 277-284, 2002.

[14] Y. Jia, Y. Zhao, T. Kusakizako et al., "TMC1 and TMC2 proteins are pore-forming subunits of mechanosensitive ion channels," Neuron, vol. 105, no. 2, pp. 310-321.e3, 2020.

[15] K. Ramzan, M. Al-Owain, N. S. Al-Numair et al., "Identification of TMC1 as a relatively common cause for nonsyndromic hearing loss in the Saudi population," American Journal of Medical Genetics. Part B, Neuropsychiatric Genetics, vol. 183, pp. 172-180, 2019.

[16] H. Wang, K. Wu, J. Guan et al., "Identification of four TMC1 variations in different Chinese families with hereditary hearing loss," Molecular Genetics \& Genomic Medicine, vol. 6, no. 4, pp. 504-513, 2018.

[17] J. Qi, L. Zhang, F. Tan et al., "Espin distribution as revealed by super-resolution microscopy of stereocilia," American journal of Translational Research, vol. 12, no. 1, pp. 130$141,2020$.

[18] J. Qi, Y. Liu, C. Chu et al., “A cytoskeleton structure revealed by super-resolution fluorescence imaging in inner ear hair cells," Cell discovery, vol. 5, no. 1, 2019.

[19] Y. Liang, A. Wang, I. A. Belyantseva et al., "Characterization of the human and mouse unconventional myosin XV genes responsible for hereditary deafness DFNB3 and shaker 2," Genomics, vol. 61, no. 3, pp. 243-258, 1999.

[20] I. A. Belyantseva, E. T. Boger, and T. B. Friedman, "Myosin XVa localizes to the tips of inner ear sensory cell stereocilia and is essential for staircase formation of the hair bundle," Proceedings of the National Academy of Sciences of the United States of America, vol. 100, pp. 13958-13963, 2003.

[21] I. A. Belyantseva, E. T. Boger, S. Naz et al., "Myosin-XVa is required for tip localization of whirlin and differential elongation of hair-cell stereocilia," Nature Cell Biology, vol. 7, no. 2, pp. 148-156, 2005.

[22] Z. Fattahi, A. E. Shearer, M. Babanejad et al., "Screening for MYO15A gene mutations in autosomal recessive nonsyndromic, GJB2 negative Iranian deaf population," American Journal of Medical Genetics. Part A, vol. 158A, no. 8, pp. 1857-1864, 2012.

[23] J. Zhang, J. Guan, H. Wang et al., "Genotype-phenotype correlation analysis of MYO15A variants in autosomal recessive 
non-syndromic hearing loss," BMC Medical Genetics, vol. 20, no. 1, p. $60,2019$.

[24] X. Wang, L. Wang, H. Peng, T. Yang, and H. Wu, “A novel p.G141R mutation in ILDR1 leads to recessive nonsyndromic deafness DFNB42 in two Chinese Han families," Neural plasticity, vol. 2018, Article ID 7272308, 6 pages, 2018.

[25] L. He, X. Pang, H. Liu, Y. Chai, H. Wu, and T. Yang, "Targeted next-generation sequencing and parental genotyping in sporadic Chinese Han deaf patients," Clinical Genetics, vol. 93, no. 4, pp. 899-904, 2018.

[26] S. Richards, on behalf of the ACMG Laboratory Quality Assurance Committee, N. Aziz et al., "Standards and guidelines for the interpretation of sequence variants: a joint consensus recommendation of the American College of Medical Genetics and Genomics and the Association for Molecular Pathology," Genetics in Medicine, vol. 17, no. 5, pp. 405423, 2015.

[27] M. B. Saï, M. Hmani-Aifa, I. Amar et al., "High frequency of the p.R34X mutation in the TMC1 gene associated with nonsyndromic hearing loss is due to founder effects," Genetic Testing and Molecular Biomarkers, vol. 14, no. 3, pp. 307-311, 2010.

[28] A. Tlili, I. B. Rebeh, M. Aifa-Hmani et al., "TMC1 but not TMC2 is responsible for autosomal recessive nonsyndromic hearing impairment in Tunisian families," Audiology \& Neuro-Otology, vol. 13, no. 4, pp. 213-218, 2008.

[29] M. Alobathani, A. Al Mutery, W. K. E. A. Mohamed, and A. Tlili, "Identification of nonsense mutation in TMC1 gene inducing hearing loss by clinical exome sequencing," Journal of Biological Sciences and Medicine, vol. 4, pp. 1-6, 2018.

[30] M. Y. Chang, C. Lee, J. H. Han et al., "Expansion of phenotypic spectrum of MYO15A pathogenic variants to include postlingual onset of progressive partial deafness," BMC Medical Genetics, vol. 19, no. 1, p. 29, 2018.

[31] M. Miyagawa, S. Y. Nishio, M. Hattori et al., "Mutations in theMYO15AGene are a significant cause of nonsyndromic hearing Loss," Annals of Otology, Rhinology \& Laryngology, vol. 124, 1_supplement, pp. 158S-168S, 2015.

[32] O. K. Egilmez and M. T. Kalcioglu, "Genetics of nonsyndromic congenital hearing loss," Scientifica, vol. 2016, Article ID 7576064, 9 pages, 2016.

[33] X. Yu, Y. Lin, J. Xu et al., "Molecular epidemiology of Chinese Han deaf patients with bi-allelic and mono-allelic GJB2 mutations," Orphanet Journal of Rare Diseases, vol. 15, no. 1, p. 29, 2020.

[34] M. B. Petersen and P. J. Willems, "Non-syndromic, autosomalrecessive deafness," Clinical Genetics, vol. 69 , no. 5, pp. 371392, 2006.

[35] S. Sang, J. Ling, X. Liu et al., "Proband whole-exome sequencing identified genes responsible for autosomal recessive non-syndromic hearing loss in 33 Chinese nuclear families," Frontiers in Genetics, vol. 10, p. 639, 2019.

[36] S. I. Kitajiri, R. McNamara, T. Makishima et al., "Identities, frequencies and origins of TMC1 mutations causing DFNB7/B11 deafness in Pakistan," Clinical Genetics, vol. 72, no. 6, pp. 546550, 2007.

[37] A. H. Chishti, A. C. Kim, S. M. Marfatia et al., "The FERM domain: a unique module involved in the linkage of cytoplasmic proteins to the membrane," Trends in Biochemical Sciences, vol. 23, no. 8, pp. 281-282, 1998.
[38] J. Li, Y. He, M. L. Weck, Q. Lu, M. J. Tyska, and M. Zhang, "Structure of Myo7b/USH1C complex suggests a general PDZ domain binding mode by MyTH4-FERM myosins," Proceedings of the National Academy of Sciences of the United States of America, vol. 114, no. 19, pp. E3776-E3785, 2017.

[39] D. W. Anderson, F. J. Probst, I. A. Belyantseva et al., "The motor and tail regions of myosin XV are critical for normal structure and function of auditory and vestibular hair cells," Human Molecular Genetics, vol. 9, no. 12, pp. 1729-1738, 2000. 